\title{
Nutritional Medicine and Diet Care
}

BRIEF REPORT

\section{Validation of a Mediterranean Diet Scoring System for Intervention Based Research}

\author{
Reeve $\mathrm{EH}^{1}$, Picicci $F^{2}$ and Deborah L Feairheller, PhD, FACSM ${ }^{2 *}$ \\ ${ }^{1}$ Department of Human Physiology, University of Oregon, USA \\ ${ }^{2}$ Department of Kinesiology, University of New Hampshire, USA \\ *Corresponding author: Deborah L Feairheller, PhD, FACSM, USAW-L1, Department of Kinesiology, University of New \\ Hampshire, 124 Main Street, Durham, NH 03824, USA, Tel: 603-862-3282
}

\begin{abstract}
Background: A Mediterranean diet pattern is cardioprotective and positively correlated with lower chronic disease risk. Certain vulnerable populations would benefit greatly from a Mediterranean diet to reduce cardiovascular disease risk, and clinical diet intervention research typically uses diet recall questionnaires. This type of dietary tracking leads to assumptions and not an actual ability to accurately track Mediterranean diet adherence in the short-term.

Objective: Thus, the purpose of this study is to test the validity of a new Mediterranean diet scoring system (MDSS) which scores diets on a weekly basis based on serving numbers within food groups.

Results: We evaluated the validity of this new MDSS to another well-established system. We analyzed 354 weeks of diet using both the new and old MDSSs, performing analysis on the specificity and sensitivity of the new MDSS to the established MDSS.

Conclusion: We found that an a priori defined adherence value of $70 \%$ using the new MDSS was both specific and sensitive to the established MDSS, comparable to other studies. In sum, this new MDSS is effective at measuring adherence to a Mediterranean diet weekly and warrants use in future studies, especially intervention-based research.
\end{abstract}

\section{Keywords}

Mediterranean diet score, Dietary tracking, Firefighter diet, Diet intervention

\section{Introduction}

Evidence suggests that diet plays a pivotal role to maintaining health, so much so that a healthy diet has been linked to outcomes involved in reducing disease risk. The Mediterranean diet has been shown to have cardioprotective benefits, and research has shown that a stronger adherence is inversely correlated with early mortality rates [1]. The Mediterranean diet is one of the best diets for maintaining cardiovascular health, and because of this, there have been an increase in the number of clinical trials utilizing the Mediterranean diet to measure health outcomes. In fact, it is thought that this diet can improve cardiovascular health like the benefits seen with the DASH diet [2].

Many studies correlate diet adherence to their main outcome, thus creating a need for an effective way of determining an individual's adherence level. Research has focused on analyzing diets through a score or a single index which results from a combination of certain included food groups. While there is no established standard for tracking Mediterranean diet adherence, many research groups have created and validated their own ways of tracking a participant's diet adherence [3]. Most of this research consists of longitudinal or crosssectional studies and aims to understand generalized diet adherence through extensive food frequency questionnaires. Many of the scores use components selected using a priori knowledge. What is lacking, however, is a Mediterranean diet scoring system (MDSS) designed to acutely measure an individual's Mediterranean diet adherence over short-term. A scoring system of this sort would greatly improve the ability to track diet adherence during a clinical trial, diet intervention, or any short-term study that requires accurate measurement of diet adherence. Furthermore, a point based MDSS would also enable certain individuals or populations the ability to monitor

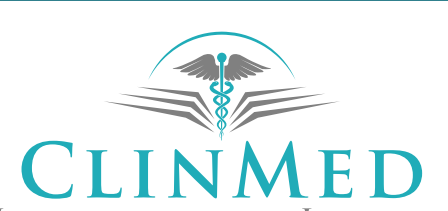

INTERNATIONAL LIBRARY 
and track diets, as needed.

Certain populations are at higher risk for cardiovascular disease. Firefighting is one of the most hazardous and dangerous jobs with an inherent increased risk of death, but the leading cause of death in this population is cardiac related [4]. Blood pressure levels are high, obesity rates are high, and fasting glucose and insulin levels are higher in firefighters compared to civilians [5]. Diets that are known to improve health, reduce inflammation, and reduce cardiac risk are important to firefighters [6]. Diet adherence is a factor that remains a limitation in many clinical studies, but a MDSS designed to accurately track adherence can better elucidate the relationship between diet and health or disease. Firefighters know that diet is important, but they don't know how to modify their diet. Many don't adhere to new dietary patterns but survey research has found that firefighters prefer the Mediterranean diet [7]. Therefore, the purpose of this study was to test the validity and sensitivity of a novel MDSS through comparison with a well-established and previously validated MDSS developed by Panagiotakos, et al. [8].

\section{Methods}

\section{Study sample}

Most of the population recruited for this study were firefighters from the Philadelphia area. The study included 59 clinically healthy adults who were not receiving treatment for cardiovascular disease, were non-smokers, and were not diabetic. All participants completed a health history questionnaire to provide information on demographic and socioeconomic variables, family history, lifestyle, and comorbidity history. Signed informed consent was collected. The protocol was approved by Institutional Review Board, and all procedures were in accordance with the ethical standards of the Helsinki Declaration. After consent, the participants were taught how to follow a modified Mediterranean diet during a training session. This session specified which foods to eat and outlined the number of serving sizes that should be consumed for each food group. All participants were provided with a study diet manual which included detailed instructions on serving sizes and portion control, sample tracking sheets, sample recipes, and information on types of foods that would fall into each food group. In addition, they were provided with colorful diet serving-size cups and a package of serving size portion-control bags. Study participants were given access to a study website where they were able to self-report serving counts for each food group during the duration of the study. The website also provided easy access to educational links, other handouts and information on the diet. All of these tools allowed for flexibility and individualization to each diet according to the person's dietary preferences and enabled them to keep their diet as close as possible to the scoring parameters. All participants completed 6 weeks of dietary tracking and self-reported weekly diets to the study personnel via the study website or by email, and all diets were analyzed using both scoring systems.

\section{Panagiotakos MDSS}

The MDSS designed by Panagiotakos, et al. outlined a scoring system containing 11 food categories, for a total of 55 points [8]. The food categories consist of non-refined cereals, potatoes, fruits, vegetables, legumes, fish, red meat and meat products, poultry, full-fat dairy products, use of olive oil in cooking, and alcoholic beverages. Each food category ranges in point value from 0-5 points. For the Panagiotakos MDSS, points are awarded via threshold values, with increasing points awarded for either frequent consumption of foods aligned with the Mediterranean diet pattern or decreased consumption of foods that stray from the diet pattern. Adherence to the diet is defined post priori as the upper tertile of scores of a given data set are deemed most adherent to the Mediterranean diet.

The MDSS developed by Panagiotakos, et al. awards points based on the number of servings consumed over a one-month period for the food groups that follow the Mediterranean diet pattern [8]. For these groups (nonrefined cereals, potatoes, fruits, vegetables, legumes, and fish) 0 points are awarded for no servings and this is scaled up to 5 points for more than 18 servings within the past month. On the other hand, food categories that deviate from a Mediterranean diet pattern (red meat and meat products, poultry, and full-fat dairy) are given the same point values in the opposite direction, where 5 points are awarded for never consuming these foods, and 0 points are scored for more than 18 servings per month.

Since the use of olive oil in cooking is encouraged in a Mediterranean pattern of eating, this MDSS also included points based on the number of times olive oil is used in cooking per week. If no olive oil is consumed, then 0 points are scored, and is scaled up to 5 points if olive oil is used "daily" in cooking. Finally, the score for alcoholic beverage consumption was measured by daily volume intake of alcoholic beverages. Since too much consumption of alcoholic beverages deviates from the Mediterranean diet pattern, a score of 5 was given for less than $300 \mathrm{ml}$ of alcoholic beverage per day and this was scaled down to 0 points if the individual consumed more than $700 \mathrm{ml}$ per day [8].

\section{Feairheller MDSS}

The new MDSS designed by our research group contains 12 food categories, for a total of 17 points (Table 1). The food categories consist of Vegetables, Fruits, Lowfat or Non-fat Dairy, Regular Dairy, Fish, Poultry, Red Meat, Beans, Nuts and Healthy Oils, Other Fats, Sweets/ Processed Foods, and Whole Grains/Potatoes. Table 2 describes the recommended intake of each food group, followed by the maximal possible points per food group. 
Table 1: Feairheller Mediterranean Diet Serving Score.

\begin{tabular}{|l|l|l|l|}
\hline & Recommendation & Calculation & Score \\
\hline Vegetables & $\geq 28$ servings/week & portion of $\mathbf{8}^{*}$ & 3 \\
\hline Fruits & $\geq 21$ servings/week & portion of $1^{*}$ & 3 \\
\hline Low-Fat or Non-Fat Dairy & $14-18$ servings/week & 0.5 if in range, otherwise 0 & 0.5 \\
\hline Regular Dairy & $\leq 6$ serving/week & 0.5 if in range, otherwise 0 & 0.5 \\
\hline Fish & $\geq 4$ servings/week & portion of $4^{*}$ & 2 \\
\hline Poultry & $\leq 3$ servings/week & 1.0 if in range, otherwise 0 & 1 \\
\hline Red Meat & $\leq 2$ servings/week & 1.0 if in range, otherwise 0 & 1 \\
\hline Beans & $\geq 3$ servings/week & 1.5 if in range, otherwise 0 & 1.5 \\
\hline Nuts \& Healthy Oils & $\geq 5$ servings/week & 2.0 if in range, otherwise 0 & 2 \\
\hline Other Fats & $\leq 7$ servings/week & 0.5 if in range, otherwise 0 & 0.5 \\
\hline Sweets \& Processed Foods & $\leq 3$ servings/week & 1.0 if in range, otherwise 0 & 1 \\
\hline Whole-Grains \& Potatoes & $\geq 49$ servings/week & portion of $49^{*}$ & 1 \\
\hline \multicolumn{1}{|c|}{ Total Score } & & & 17 \\
\hline
\end{tabular}

"Portion example: If 19 servings are consumed out of 28 recommended at 3 pt possible score, $19 / 28=0.67 \times 3=2.03$ pts earned. "Maximal points possible per food group.

Table 2: Population demographics and clinical characteristics.

\begin{tabular}{|l|l|}
\hline & Value \\
\hline Age, years & $42.7(13.6)$ \\
\hline Sex, Male/Female & $44 / 15$ \\
\hline Population, Civilians/Firefighters & $24 / 35$ \\
\hline Family History, Yes/No & $33 / 26$ \\
\hline Hypertensive, $n(\%)$ & $35(59.3)$ \\
\hline Hyperlipidemic, $n(\%)$ & $29(49.2)$ \\
\hline Physical Activity, Minutes of exercise per week & $102.5(101.3)$ \\
\hline
\end{tabular}

*Data are presented as $\mathrm{N}$ or Mean (SD).

Food groups are weighted to award higher point values for adhering to certain food group recommendations over others. The weighted food groups are a key feature of this new MDSS, as this new system strives to accurately assess not only an individual's dietary pattern, but how adherent they are to the most important elements of the Mediterranean diet. This new MDSS uses an a priori defined adherence value of $70 \%$ or more of the total score ( $\geq 11.9$ out of 17 points).

The new MDSS assigns point value for the Vegetables, Fruit, Fish, and Whole Grains \& Potatoes categories on a sliding scale based on percentage. The number of servings consumed compared to the recommended number will be translated to a point value based on percentage and maximal possible points. For example, and individual who consumes 19 servings of vegetables would score 2.03 points out of a possible $3(19 / 28=$ $0.67 \times 3=2.03 \mathrm{pts}$ earned). For all the other food group categories, scoring points are awarded on a met or not met criteria based on the maximal point value. If the individual consumes the recommended number of servings, they are scored maximal points for that category, otherwise a score of 0 is given.
Arguably, the most important aspect of this scoring system is the weighted point values for food categories. Studies have shown that increased fruit and vegetable intake may reduce cardiovascular risk when consumed in a normal diet [9]. Additionally, an increased fish intake can reduce cardiovascular disease risk in a population of high-risk individuals [10]. Beans and healthy oils, specifically olive oil, show cardioprotective benefits and therefore are included in the Mediterranean diet $[11,12]$. For these reasons, we have assigned these four food categories a higher weight. Furthermore, a meta-analysis confirms that whole-grain consumption can reduce the risk of many chronic diseases and early mortality which led us to create percentage-based scoring for that group [13]. Since we believe higher consumption of these foods aligns more appropriately with a Mediterranean diet pattern, an enhanced method of tracking adherence to these food groups was necessary.

\section{Statistical analysis}

Statistical analysis was performed using SPSS version 24.0 (SPSS Inc., Chicago, IL, USA). Data were analyzed using descriptive statistical methods. Results are reported as means and standard deviation for quantitative variables and as frequencies for qualitative variables. Pearson correlation coefficient was used as the measure of association. To validate the efficacy of the new MDSS we compared our new scoring system to the previously established system by Panagiotakos, et al. (2006) and evaluated our new MDSS by conducting specificity and sensitivity analysis previously described by Sofi, et al. with their validation of the MEDI-LITE scoring system $[8,14]$. We obtained 354 weeks of individual diets and scored them for adherence using the Panagiotakos MDSS and our new MDSS. We used 
our a priori defined adherence value of $70 \%$ of the total score. The sensitivity of our MDSS was defined as the ratio of true positives to true positives + false negatives [14]. Specificity was defined as the ratio of true negatives to true negatives + false positives. A true positive was defined as score that met adherence values for both scoring methods, followed by a true negative in which a score did not meet adherence values for either scoring method. A false negative was defined as a score that met adherence only for Panagiotakos MDSS, while a false positive was a score that met adherence only for the new MDSS. Additionally, the receiver operating characteristics (ROC) was calculated as, the sensitivity * (1 - sensitivity).

\section{Results}

Table 2 shows the demographic characteristics of the study population. The participants were mostly from the suburban Philadelphia PA area (55/59, 93\%). The population was educated with 19 reporting graduate degree, 20 reporting undergraduate degree, 13 reporting associates degree, and 7 reported high school education. Overall, the Feairheller MDSS was correlated with the Panagiotakos MDSS (Figure 1).

When comparing 354 weeks of individual diets, we found that our new MDSS is both sensitive and specific to the previous MDSS designed by Panagiotakos, et al., [8]. When comparing diet scores, we found the number of true positives to be 114 , true negatives to be 175 , False negatives to be 20 , and false positives to be 45 . Our sensitivity analysis reveals the new MDSS to be $85.1 \%$ sensitive to the old scoring system. Our specificity analysis reveals the new MDSS to be $79.5 \%$ specific to the old scoring system. The ROC was 0.174 .

\section{Discussion}

We validated a new MDSS by comparing to a previously established and validated MDSS developed by Panagiotakos, et al. The a priori defined adherence ( $\geq 70 \%$ of total points) is both sensitive, specific, and correlative to previous MDSS. We determined that for our MDSS, post-priori adherence scores based on upper tertial of the population was both less sensitive and specific. Additionally, a priori defined adherence scores give individuals a reasonable goal to achieve and can be especially helpful for intervention-based studies relying on diet adherence. Our new MDSS is literature-based, uses 12 weighted food categories, and scores a total of 17 possible points. The new MDSS created by our group is designed to be used in intervention-based research to acutely measure adherence to a Mediterranean diet on a weekly basis.

Previous MDSS have also used sensitivity, specificity, and ROC values to validate a diet scoring system [14]. Our sensitivity and specificity analyses display results similar to that of the previously described MDSS indexes. Recently, Mila-Vallarroel, et al. reported a correlation coefficient threshold of 0.70 to indicated high correlation between different Mediterranean diet indices [15]. Our scoring system met this threshold with a correlation coefficient of 0.6996 in relation to the MDSS created by Panagiotakos, who pioneered the idea of a scoring system to assess individual's adherence to a Mediterranean diet pattern.

Our new MDSS contains novel advancements which would warrant it's use in future studies. One aspect of this MDSS is the inclusion of weighted food category scores. Unlike many others, this scoring system gives higher weight, in the form of point values, to food

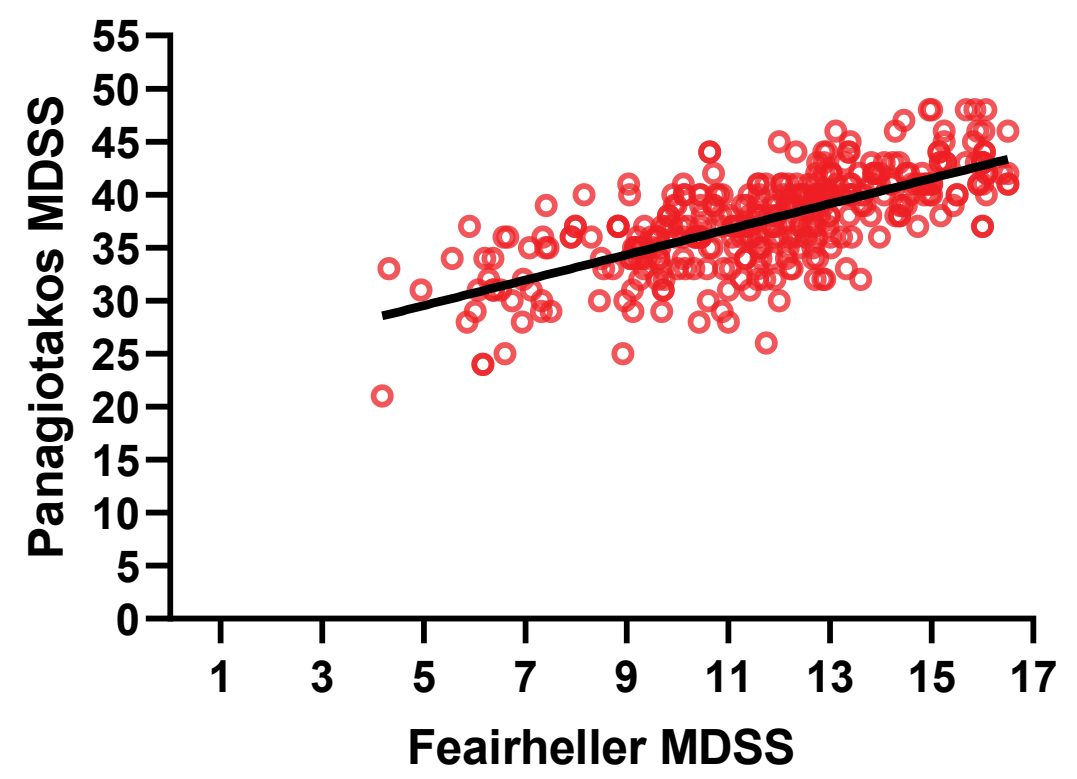

Figure 1: Correlation between weekly Feairheller Mediterranean Diet Score System (MDSS) score and Panagiotakos MDSS score.

$R=0.6996 ; p<0.000$. 
categories that align strongest with the ideals of a Mediterranean diet pattern. Food categories like fruits, vegetables, fish and healthy oils contain the highest point values because adherence to these aspects of the diet is a better representation of a Mediterranean dietary pattern. With our new scoring system, partial 'credit' is earned based on number of servings consumed in food groups. This type of scoring could improve motivation and may increase adherence. It has been reported that olive oil, fruits and vegetables are the food groups most correlated among various Mediterranean diet indexes designed to assess adherence, and these components have stronger emphasis with our MDSS [16].

Another novel aspect of this scoring system is its ability to acutely measure Mediterranean diet adherence. Other previously established scoring systems are practical for epidemiological, longitudinal, and cross-sectional studies as most use food frequency questionnaires to gather an estimation of an individual's diet across time [16]. With growing evidence supporting the benefits of a Mediterranean diet, there is a growing need for a way to track adherence to the Mediterranean diet on a short-term basis. Weekly scoring gives immediate feedback and diet intervention-based studies would benefit from using this scoring system as it is designed to assist with tracking an individual's diet adherence over short term.

In sum, the results of this study report that our new weekly MDSS is well correlated with established MDSS used by Panagiotakos, et al. which scores diets based on frequency of consumption over a month-long period [8]. This new scoring method allows immediate and weekly feedback on dietary adherence which is based on a traditional Mediterranean reference pattern of food grouping. Further studies are needed using this scoring system.

\section{Declaration}

The authors declare that they have no conflict of interest.

\section{Acknowledgements}

The authors thank all the study participants, including the firefighters from the fire departments in the Philadelphia suburban area for their participation in the study. The authors also thank all the HEART lab research students who helped with data collection and dietary tracking.

\section{Funding}

This work was supported by the American Heart Association Grant 19AIREA34450151(Feairheller).

\section{Authors' Contributions}

All authors contributed to writing of the manuscript. FP contributed to data analysis. ER and DF completed data collection and confirmed data analysis.

\section{References}

1. Estruch R, Ros E, Salas-Salvado J, Covas MI, Corella D, et al. (2013) Primary prevention of cardiovascular disease with a Mediterranean diet. NEJM 368: 1279-1290.

2. Park YM, Steck SE, Fung TT, Zhang J, Hazlett LJ, et al. (2017) Mediterranean diet, Dietary Approaches to Stop Hypertension (DASH) style diet, and metabolic health in U.S. adults. Clin Nutr 36: 1301-1309.

3. Hernández-Ruiz A, García-Villanova B, Guerra Hernández EJ, Amiano P, Azpiri M, et al. (2015) Description of indexes based on the adherence to the Mediterranean dietary pattern: A Review. Nutr Hosp 32: 1872-1884.

4. Soteriades ES, Smith DL, Tsismenakis AJ, Baur DM, Kales SN (2011) Cardiovascular disease in US firefighters: A systematic review. Cardiol Rev 19: 202-215.

5. Soteriades ES, Hauser R, Kawachi I, Liarokapis D, Christiani DC, et al. (2005) Obesity and cardiovascular disease risk factors in firefighters: A prospective cohort study. Obes Res 3: 1756-1763.

6. Lopez-Garcia E, FB Hu (2004) Nutrition and the endothelium. Curr Diab Rep 4: 253-259.

7. Yang J, Farioli A, Korre M, Kales SN (2015) Dietary preferences and nutritional information needs among career firefighters in the United States. Glob Adv Health Med 4: 16-23.

8. Panagiotakos DB, Pitsavos C, Stefanadis C (2006) Dietary patterns: A Mediterranean diet score and its relation to clinical and biological markers of cardiovascular disease risk. Nutr Metab Cardiovasc Dis 16: 559-568.

9. Alissa EM, Ferns GA (2017) Dietary fruits and vegetables and cardiovascular diseases risk. Crit Rev Food Sci Nutr 57: 1950-1962.

10. Mohan D, Mente A, Dehghan M, Rangarajan S, O'Donnell $M$, et al. (2021) Associations of fish consumption with risk of cardiovascular disease and mortality among individuals with or without vascular disease from 58 countries. JAMA Intern Med 181: 631-649.

11. Guasch-Ferré M, Hu FB, Martínez-González MA, Fitó $M$, Bulló $M$, et al. (2014) Olive oil intake and risk of cardiovascular disease and mortality in the PREDIMED Study. BMC Med 12: 78.

12. Mullins AP, Arjmandi BH (2021) Health benefits of plantbased nutrition: Focus on beans in cardiometabolic diseases. Nutrients 13: 519.

13. Aune D, Keum N, Giovannucci E, Fadnes LT, Boffetta $P$, et al. (2016) Whole grain consumption and risk of cardiovascular disease, cancer, and all cause and cause specific mortality: Systematic review and dose-response meta-analysis of prospective studies. BMJ 353: i2716.

14. Sofi F, Dinu M, Pagliai G, Marcucci R, Casini A (2017) Validation of a literature-based adherence score to Mediterranean diet: The MEDI-LITE score. Int J Food Sci Nutr 68: 757-762.

15. Mila-Villarroel R, Bach-Faig A, Puig J, Puchal A, Farran A, et al. (2011) Comparison and evaluation of the reliability of indexes of adherence to the Mediterranean diet. Public Health Nutr 14: 2338-2345.

16. Bach A, Serra-Majem L, Carrasco JL, Roman B, Ngo J, et al. (2001) The use of indexes evaluating the adherence to the Mediterranean diet in epidemiological studies: A review. Public Health Nutr 9: 132-146. 